\title{
Fibronectin induces epithelial-mesenchymal transition in human breast cancer MCF-7 cells via activation of calpain
}

\author{
CHENG-LIN LI ${ }^{1 *}$, DAN YANG $^{1 *}$, XIN CAO $^{1}$, FAN WANG $^{1}$, \\ DUAN-YANG HONG ${ }^{2,3}$, JING WANG ${ }^{2,3}$, XIANG-CHUN SHEN ${ }^{2,3}$ and YAN CHEN ${ }^{2,3}$
}

\author{
${ }^{1}$ Jiangsu Key Laboratory of New Drug Research and Clinical Pharmacy, Jiangsu Center for The Collaboration and Innovation \\ of Cancer Biotherapy, Xuzhou Medical University, Xuzhou, Jiangsu 221004; \\ ${ }^{2}$ Key Laboratory of Optimal Utilization of Natural Medicine Resources, Guizhou Medical University; \\ ${ }^{3}$ Department of Pharmacology of Chinese Material Medica, Guizhou Medical \\ University, Guiyang, Guizhou 550025, P.R. China
}

Received January 31, 2016; Accepted January 17, 2017

DOI: $10.3892 / \mathrm{ol} .2017 .5896$

\begin{abstract}
Fibronectin (FN) is a primary component of the mammary mesenchymal compartment and undergoes dramatic changes during breast cancer development. Increased $\mathrm{FN}$ expression is associated with an invasive and metastatic breast cancer phenotype. The present study demonstrated that FN causes an epithelial-mesenchymal transition (EMT)-like morphological change in MCF-7 breast cancer cells. FN stimulation caused the downregulation of epithelial markers E-cadherin and tight junction protein ZO-1, and the upregulation of mesenchymal markers $\mathrm{N}$-cadherin and vimentin. Additionally, FN promoted cell migration and invasion in MCF-7 cells, with increased expression of calpain-2 and proteolysis of focal adhesion kinase 1 (FAK), indicating calpain activation. Notably, the FN induced changes in morphology and EMT markers were reversed with the treatment of calpain-specific inhibitors, calpain inhibitor I (N-acetyl-L-leucyl-L-leucyl-L-norleucinal), calpeptin and calpain inhibitor IV. Meanwhile, the effects of FN on cell migration and invasion, as well as FAK proteolysis were markedly suppressed by calpain inhibitors. Taken together, the results of the present study indicate that calpain plays an essential role in FN-induced EMT response, and that targeting calpain signaling may be a potential strategy to reduce breast cancer metastasis.
\end{abstract}

Correspondence to: Professor Yan Chen or Professor Xiang-Chun Shen, Key Laboratory of Optimal Utilization of Natural Medicine Resources, Guizhou Medical University, 9 Beijing Road, Guiyang, Guizhou 550025, P.R. China

E-mail: s0710189@sina.com

E-mail: shenxiangchun@126.com

${ }^{*}$ Contributed equally

Key words: fibronectin, calpain, epithelial-mesenchymal transition, migration, invasion, breast cancer

\section{Introduction}

The extracellular matrix (ECM) is composed of highly variable and dynamic components, which regulate cell behavior and fate (1). In breast cancer, a number of ECM proteins are significantly deregulated and specific matrix components promote tumor progression and metastasis (2). Fibronectin (FN) is a component of the mammary mesenchymal compartment. FN is not expressed in normal adult breast tissue, whereas increased mRNA and protein levels have been reported in the stroma of breast tumors (3). A high level of FN expression is associated with an increased risk of mortality of breast cancer, and it may be a useful marker for predicting poor prognosis in breast cancer patients (4). In addition, increased FN expression is associated with an invasive and metastatic breast cancer phenotype (5). Changes in the production and organization of FN contribute to the 'pre-metastatic niche', which facilitates the adhesion of bone marrow-derived cells to promote tumor progression and metastasis $(6,7)$.

An important event in the initiation of cancer metastasis is epithelial-mesenchymal transition (EMT), which is a process during which epithelial cells lose apical-basal polarity and gain a mesenchymal phenotype (8). During this transition, epithelial carcinoma cells acquire phenotypic changes and become highly motile and invasive, which facilitates the migration of cells from the originating site of the tumor to distal sites (9). Recent research demonstrates that FN induces an EMT response in MCF-10A human mammary epithelial cells, via cooperation with Src kinase and extracellular signal-regulated kinase (ERK)/mitogen-activated protein kinase signaling, which is initiated by the type-I transforming growth factor- $\beta$ (TGF- $\beta$ ) receptor (10). However, the detailed mechanisms that enable an EMT response in breast cancer cells with aberrant FN stimulation remain to be elucidated.

The calpain family is a group of calcium-dependent cysteine proteases and is involved in a variety of biological activities by limited proteolysis of numerous substrates. Two members of the calpain family $\mu$-calpain (calpain-1) and m-calpain (calpain-2) are ubiquitously expressed (11). Altered activity and expression of calpains has been implicated in a number 
of disease states, including cancer (12). Investigations have demonstrated that calpain has a role in breast cancer progression, prognosis and treatment response, thus it is considered as a potential anticancer target (13). Furthermore, calpain activity is aberrantly higher in breast cancer tissues compared with normal breast tissues, and its expression correlates with metastatic phenotypic characteristics and increased invasive properties of tumors (14-16). Calpain also plays an essential role in regulating cell migration and invasion by promoting focal adhesion and invadopodia/podosome disassembly through cleavage of its substrates, including talin (17), focal adhesion kinase (FAK) (18), cortactin (19) and ezrin (20). In addition, calpain is one of the key downstream molecules required for growth factor-induced motility in breast cancer (21). Notably, calpain is also implicated in the EMT process in cancer cells. It has been previously reported that calpain 2 is upregulated during TGF- $\beta$-induced EMT in A549 lung adenocarcinoma cells (22). In addition, FN may induce cell migration and invasion in A549 cells via ERK1/2-calpain-2 signaling (23), indicating a potential role for calpain in FN induced cellular response.

In view of the previously reported notable effects of calpain on cell migration and invasion in breast cancer, the present study sought to identify whether the upregulation and activation of calpain play a role in $\mathrm{FN}$ induced migration and invasion in breast cancer cells. This will enable a further understanding of the role of calpain in the process of FN-induced EMT.

\section{Materials and methods}

Reagents and antibodies.FN(Sigma-Aldrich; Merck Millipore, Darmstadt, Germany) was dissolved in sterile distilled $\mathrm{H}_{2} \mathrm{O}$ as a stock solution at $1 \mathrm{mg} / \mathrm{ml}$ and stored at $-20^{\circ} \mathrm{C}$. Calpeptin (N-benzyloxycarbonyl-L-leucylnorleucinal) and calpain inhibitor IV (Calbiochem; Merck Millipore) were dissolved in dimethylsulfoxide (DMSO; $0.1 \mathrm{M}$ ) and stored at $-20^{\circ} \mathrm{C}$ as stock solutions. Calpain inhibitor I (ALLN; Calbiochem; Merck Millipore) was dissolved in DMSO $(0.1 \mathrm{M})$ and stored at $4^{\circ} \mathrm{C}$. Matrigel was purchased from BD Biosciences (San Jose, CA, USA). Antibodies to calpain-1 (catalog no. sc-13990; 1:500; polyclonal rabbit anti-human), calpain-2 (catalog no. sc-373966; 1:500; polyclonal rabbit anti-human) and FAK (catalog no. sc-557; 1:500; polyclonal rabbit anti-human) were purchased from Santa Cruz Biotechnology, Inc. (Dallas, TX, USA). Antibodies to E-cadherin (catalog no. 3195; 1:1,000; polyclonal rabbit anti-human), ZO-1 (catalog no. 8193; 1:1,000; polyclonal rabbit anti-human), N-cadherin (catalog no. 13116; 1:1,000; polyclonal rabbit anti-human) and vimentin (catalog no. 5741; 1:1,000; polyclonal rabbit anti-human, ) were obtained from Cell Signaling Technology, Inc. (Danvers, MA, USA). Antibody to $\beta$-actin (catalog no. AP0060; 1:3,000; polyclonal rabbit anti-human) was purchased from Bioworld Technology, Inc. (St. Louis Park, MN, USA).

Cell lines and cell culture. The MCF-7 human breast cancer cell line was purchased from the Cell Bank at the Shanghai Institute of Cell Biology (Shanghai, China). The cells were cultured in Dulbecco's modified Eagle's medium (DMEM; Gibco; Thermo Fisher Scientific, Inc., Waltham, MA, USA) at $37^{\circ} \mathrm{C}$ in a humidified incubator $\left(5 \% \mathrm{CO}_{2}\right)$ and supplemented with $10 \%$ fetal bovine serum (FBS; Gibco; Thermo Fisher Scientific, Inc.), $100 \mathrm{U} / \mathrm{ml}$ penicillin (Beyotime Institute of Biotechnology, Haimen, China) and $100 \mu \mathrm{g} / \mathrm{ml}$ streptomycin (Beyotime Institute of Biotechnology).

Cell treatment. Cells were replated on $\mathrm{FN}(20 \mu \mathrm{g} / \mathrm{ml})$ for $48 \mathrm{~h}$ prior to analysis. When inhibitors were used, cells were incubated in medium at $37^{\circ} \mathrm{C}$ containing the inhibitors for $1 \mathrm{~h}$ prior to being replated on FN. The inhibitors were used as follows: ALLN $(10 \mu \mathrm{M})$, calpeptin $(50 \mu \mathrm{M})$ and calpain inhibitor IV $(25 \mu \mathrm{M})$.

Wound healing assay. The wound healing assay is a conventional method used to study directional cell migration in vitro. Cells were seeded into 6-well plates and grown to $90 \%$ confluence. The monolayers were scraped with a micropipette tip and rinsed with phosphate-buffered saline three times to remove any floating cells. Representative images were captured at 0 and $48 \mathrm{~h}$ after scraping (IX53; Olympus Corporation, Tokyo, Japan) and analyzed (cellSens; version 1.14.14116.2; Olympus Corporation). The level of cell migration was quantified as a percentage compared with the cells at $0 \mathrm{~h}$ of each group. The data shown were obtained from three independent experiments.

Invasion assay. Invasive ability of the cells was measured by using a Transwell chamber (EMD Millipore, Billerica, MA, USA) containing membranes with pores $(8 \mu \mathrm{m})$, which were initially coated with Matrigel (40 $\mu \mathrm{g} / 100 \mu \mathrm{l} /$ chamber) as previously described (24). Following treatment with FN for $48 \mathrm{~h}$ or ALLN $(10 \mu \mathrm{M})$, calpeptin $(50 \mu \mathrm{M})$ and calpain inhibitor IV $(25 \mu \mathrm{M})$ for $1 \mathrm{~h}$ prior to treatment with $\mathrm{FN}$, the cells were suspended in serum-free medium $\left(5 \times 10^{5}\right.$ cells $\left./ \mathrm{ml}\right)$ and seeded into the upper compartment, while the DMEM containing 10\% FBS was added in the lower compartment as a chemo-attractant. Following incubation at $37^{\circ} \mathrm{C}$ for $24 \mathrm{~h}$, the non-invasive cells on the upper side of the membrane were removed with a cotton swab. The invasive cells on the lower surface were fixed with $100 \%$ methanol and stained with $0.5 \%$ crystal violet at room temperature for $20 \mathrm{~min}$ (Beyotime Institute of Biotechnology). The invasive cells were quantified by manual counting under an inverted microscope (CX51; Olympus Corporation) at x100 magnification. For each experimental group, 5 randomly selected fields were analyzed.

Western blot analysis. Cells were collected and lyzed in lysis buffer (Thermo Scientific Inc.). The lysates were clarified by centrifugation at $4^{\circ} \mathrm{C}$ for $15 \mathrm{~min}$ at $13,000 \times \mathrm{g}$. The protein concentration in the supernatants was measured using bicinchoninic acid assay kit (Thermo Scientific Inc.) with a microplate reader (ELX808IU; BioTek Instruments, Inc., Winooski, VT, USA). Total protein (30 $\mu \mathrm{g} / \mathrm{lane})$ was separated by $10 \%$ SDS-PAGE and transferred to a polyvinylidene difluoride membrane (EMD Millipore, Billerica, MA, USA). The membrane was blocked with $1 \%$ bovine serum albumin and subsequently incubated with the appropriate primary antibodies as described in the reagents and antibodies section overnight at $4^{\circ} \mathrm{C}$. The membranes were washed three times with TBS containing Tween-20 buffer and incubated with the IRDyeTM800-conjugated secondary antibody (catalog no. P/N 925-32211; 1:20,000; Rockland Immunochemicals, 

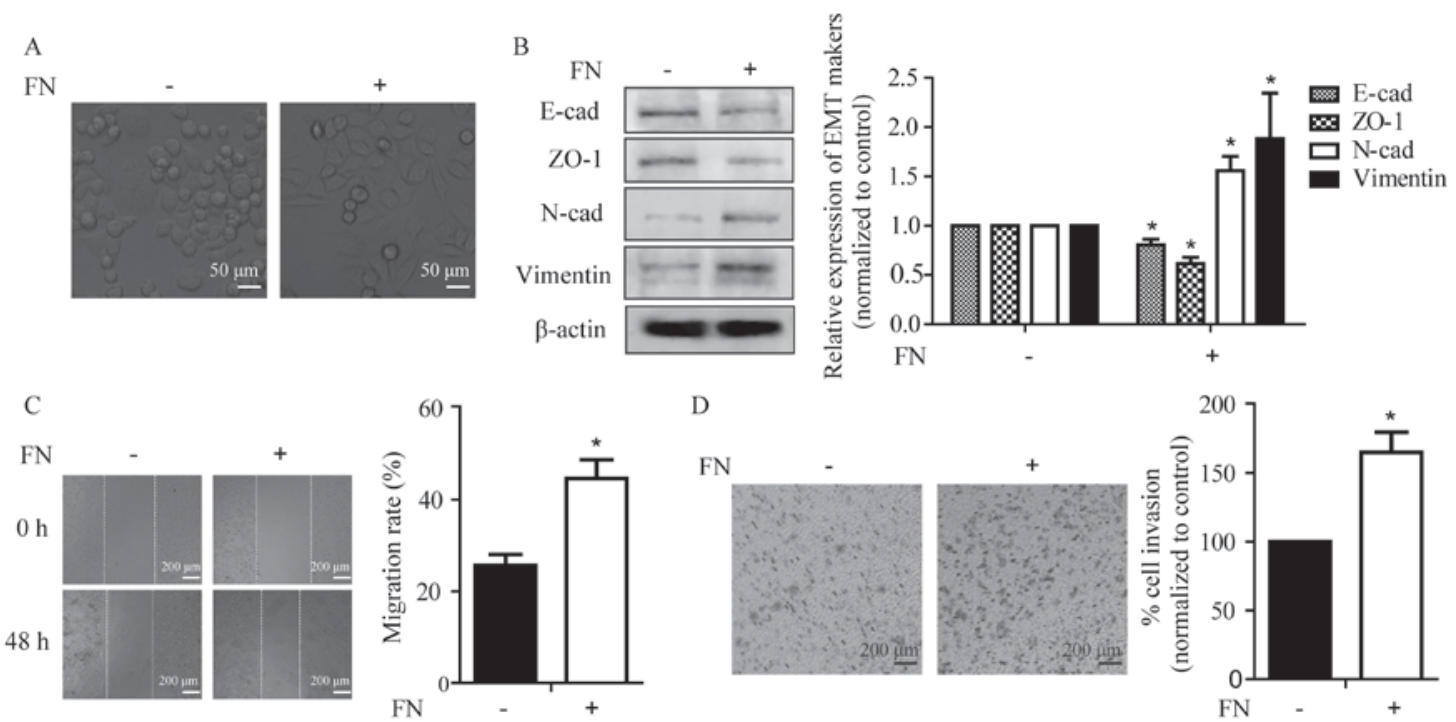

Figure 1. FN stimulates changes in cell morphology and expression of EMT markers, as well as promoting cell migration and invasion in MCF-7 cells. (A) Micrographs of MCF-7 cells untreated and treated with FN for $48 \mathrm{~h}$ (magnification, x400). (B) FN stimulated changes in the expression of EMT markers. (C) FN promoted migration of MCF-7 cells. Monolayer cells were scratched in the presence and absence of FN (20 $\mu \mathrm{g} / \mathrm{ml})$ for $48 \mathrm{~h}$. The images were captured at 0 and $48 \mathrm{~h}$ following scratching (magnification, x100). (D) FN promoted invasion of MCF-7 cells. Cells were plated with and without FN (20 $\mu \mathrm{g} / \mathrm{ml})$ for $48 \mathrm{~h}$. The invasive cells that passed through the membrane were examined with crystal violet staining. The images are representative of 3 separate experiments (magnification, $\mathrm{x} 100$ ). Values are expressed as the mean \pm standard error. "P<0.05 vs. the control. E-cad, E-cadherin; FN, fibronectin; EMT, epithelial-mesenchymal transition; N-cad, N-cadherin; ZO-1, tight junction protein ZO-1.

Inc., Pottstown, PA, USA) at $37^{\circ} \mathrm{C}$ for $1 \mathrm{~h}$, followed by washing four times with phosphate-buffered saline. Images of the membrane were captured with the Odyssey Infrared Imaging System (LI-COR Inc., Lincoln, NE, USA). $\beta$-actin was used as an endogenous control, and the relative expression of proteins was normalized to the control group. Densities of signals on blots were evaluated using ImageJ software (U.S. National Institutes of Health, Bethesda, MD, USA).

Statistical analysis. All data obtained from at least three independent experiments are expressed as the mean \pm standard error. Statistically significant differences were calculated by the Student's $t$-test for comparing between two groups and one-way analysis of variance for multiple-group comparisons, followed by the Bonferroni post-hoc test. $\mathrm{P}<0.05$ was considered to indicate a statistically significant difference.

\section{Results}

FN stimulates cell migration and invasion with changes in EMT marker expression in MCF-7 breast cancer cells. Although FN has been previously demonstrated to induce EMT in breast epithelial cells (10), whether it induces EMT in breast cancer cells requires investigation. As shown in Fig. 1A, FN was capable of inducing an EMT-like morphological change in MCF-7 cells, from a cobblestone-like epithelial morphology to a spindle-like fibroblast appearance. Corresponding with the changes in morphology, FN treatment for $48 \mathrm{~h}$ led to alterations in the expression of epithelial markers, including a significant decrease in E-cadherin and ZO-1, as well as a significant increase in mesenchymal markers $\mathrm{N}$-cadherin and vimentin in MCF-7 cells (Fig. 1B). It has been well demonstrated that EMT is associated with increased cellular motility (25). Thus, the motile phenotype of FN-induced cells was evaluated by the wound healing and Transwell invasion assays. FN treatment promoted wound closure from $25.6 \pm 2$ to $44.6 \pm 4 \%$ and invasive ability of the MCF-7 cells from 100 to $164.9 \pm 15 \%$. (Fig. 1C and D). These results indicated that FN altered the expression of EMT markers and facilitated cellular motility.

FN promotes FAK cleavage with an increased expression of calpain-2. As a central component of focal adhesions, FAK can interact with and phosphorylate several members of the focal adhesion complex through multiple protein-binding domains (26). The cleavage of FAK leads to adhesion complex turnover and increased cellular motility (27). As shown in Fig. 2A, following FN treatment for $48 \mathrm{~h}$, FAK was cleaved to the $90 \mathrm{kDa}$ form. FAK is a sensitive substrate of calpain and can be hydrolyzed to form the low molecular weight isoforms via calpain activation. Furthermore, the generated $\sim 90 \mathrm{kDa}$ cleavage fragment of FAK was identical in size to a previously identified calpain-mediated cleavage product of FAK (28). Therefore, whether the expression of calpain is changed following FN stimulation was assessed. It was observed that the expression of calpain-1 increased following FN stimulation, although this increase was not significant. The expression of calpain-2 was significantly upregulated (Fig. 2B).

Inhibition of calpain reverses FN-induced alteration of EMT markers. As shown in Figs. 1 and 2, upregulation of calpain-2 was observed in the process of FN-induced EMT in MCF-7 cells. To investigate whether activated calpain is a requisite or concomitant in FN-induced EMT, three specific calpain inhibitors, ALLN, calpeptin and calpain inhibitor IV were used. Compared with non-treated cells, FN treatment led to downregulation of E-cadherin and $\mathrm{ZO}-1$, but upregulation of $\mathrm{N}$-cadherin and vimentin. The changes of E-cadherin, ZO-1 and vimentin expression induced by FN were markedly 
A
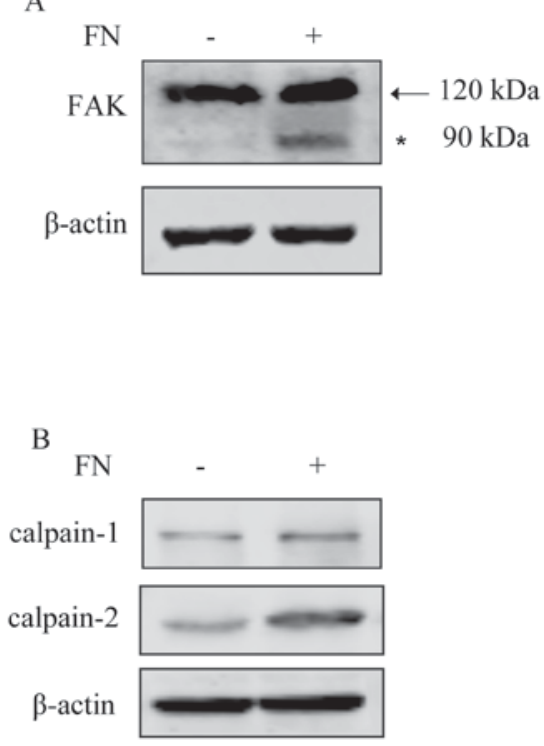
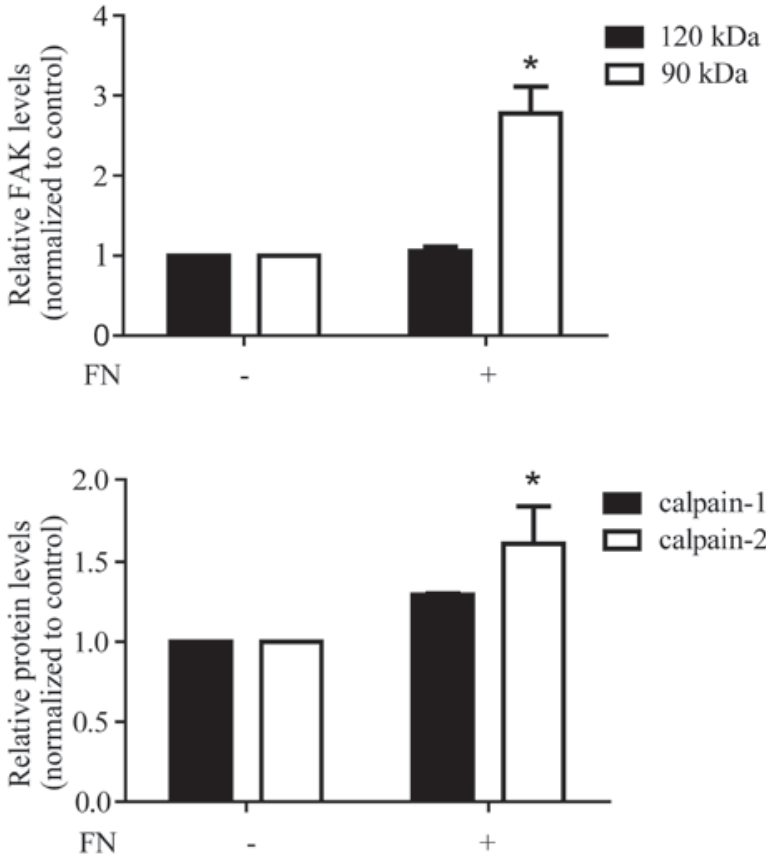

Figure 2. Impact of FN on the processing of FAK and calpain expression in MCF-7 cells. (A) FN enhanced FAK proteolysis in MCF-7 cells. Arrow indicates the wild-type (120 kDa) and "indicates the processed form(s) $(90 \mathrm{kDa})$ of FAK. (B) FN induced the upregulation of calpain-2 in MCF-7 cells. Values are expressed as the mean \pm standard error. ${ }^{*} \mathrm{P}<0.05$ vs. the control. FAK, focal adhesion kinase $1 ; \mathrm{FN}$, fibronectin.

A

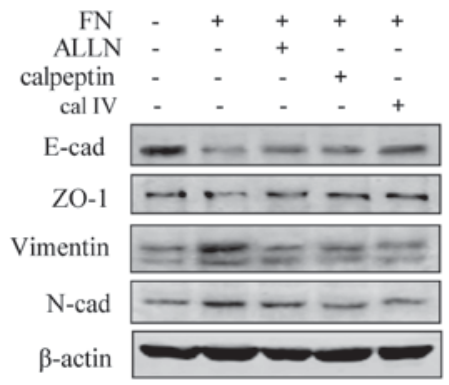

B

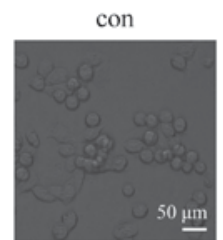

FN

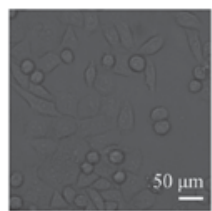

产

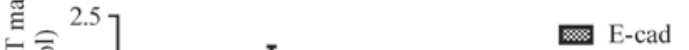

음 E-cad

开 * * * I $^{*}$ 口 $\mathrm{N}-\mathrm{cad}$

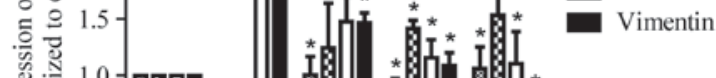

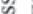

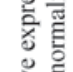

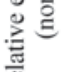

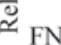

ALLN

calpeptin

cal IV
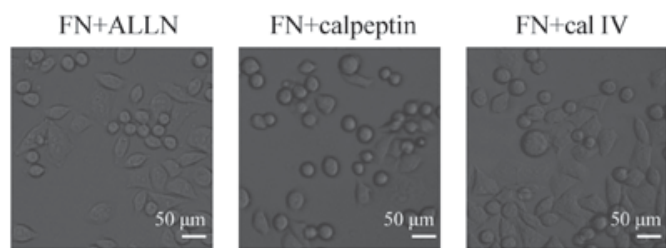

Figure 3. Calpain mediates FN-induced EMT of MCF-7 cells. Cells were pretreated with and without ALLN $(10 \mu \mathrm{M})$, calpeptin $(50 \mu \mathrm{M})$ and calpain inhibitor IV $(25 \mu \mathrm{M})$ for $1 \mathrm{~h}$ prior to treatment with FN for $48 \mathrm{~h}$. (A) Inhibition of calpain activation blocked FN-induced change in EMT marker expression. The expression of EMT markers was detected by western blotting, with $\beta$-actin as loading control. Values are expressed the mean \pm standard error. "P<0.05, FN vs. FN+ALLN, FN+calpeptin and FN+calpain inhibitor IV. (B) Repression of calpain activation inhibited FN-induced morphological changes. Morphological observation of MCF-7 cells was performed following FN treatment with or without calpain inhibitors (magnification, x400). ALLN, calpain inhibitor I; con, control; EMT, epithelial-mesenchymal transition; FN, fibronectin; cal IV, calpain IV; E-cad, E-cadherin; N-cad, N-cadherin; ZO-1, tight junction protein ZO-1.

suppressed by ALLN. Calpeptin or calpain inhibitor IV also attenuated FN-induced upregulation of E-cadherin and ZO-1, and downregulation of vimentin and $\mathrm{N}$-cadherin (Fig. 3A). In addition, calpain inhibitors calpeptin and calpain inhibitor IV also reversed the FN-induced EMT-like morphological change in MCF-7 cells (Fig. 3B).
Calpain is required for FN-induced cell migration, invasion and proteolytic cleavage of FAK. The results of the present study demonstrated that calpain inhibitors reversed $\mathrm{FN}$-induced EMT, which prompted an investigation into whether calpain inhibition affects FN-induced cell motility. MCF-7 cells were pre-incubated with or without calpain inhibitors for $1 \mathrm{~h}$ prior 

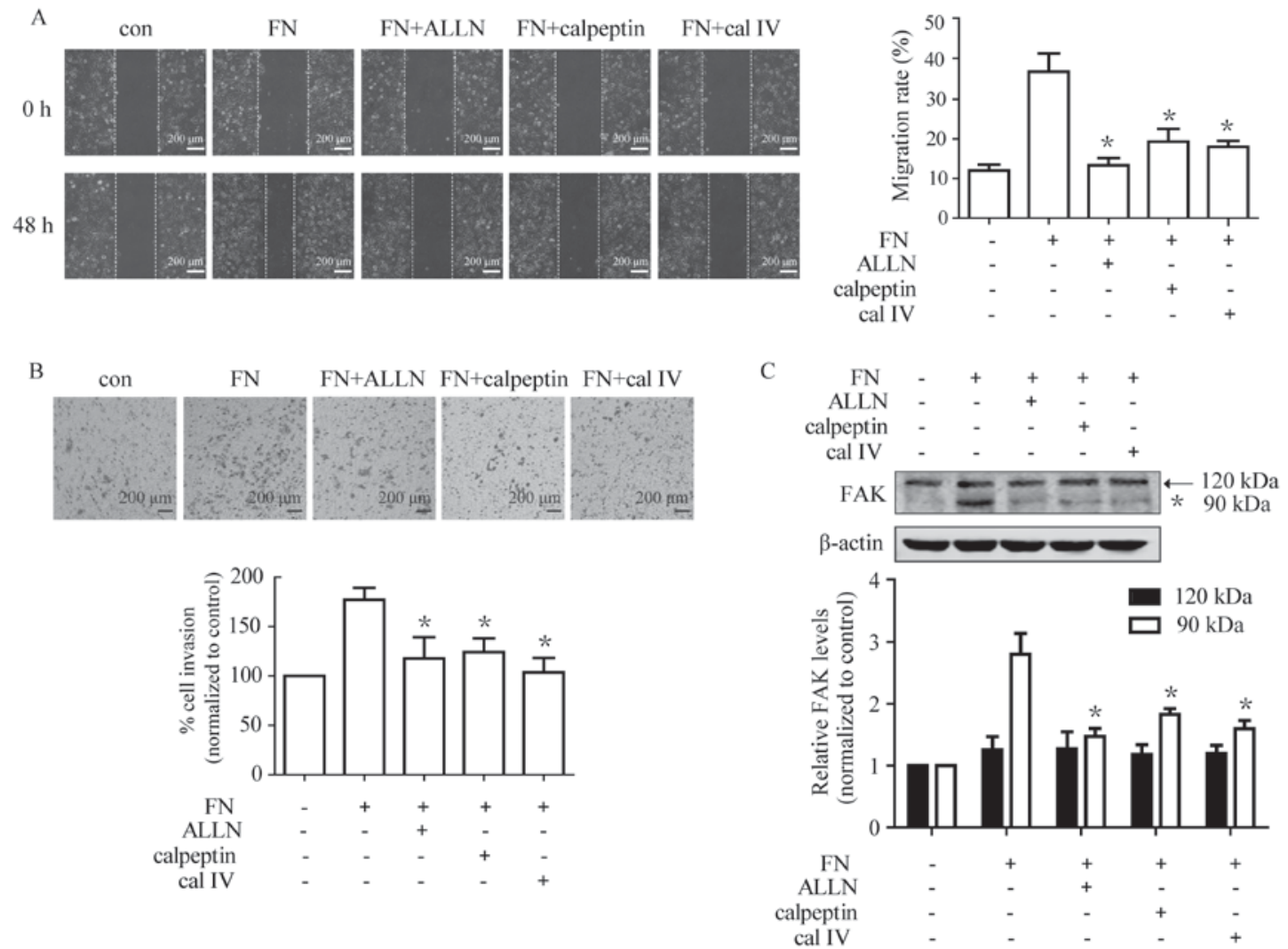

Figure 4. Calpain is involved in cell migration, invasion and FAK cleavage during FN-induced EMT in MCF-7 cells. Cells were pretreated with and without $\operatorname{ALLN}(10 \mu \mathrm{M})$, calpeptin $(50 \mu \mathrm{M})$ and calpain inhibitor IV (cal IV, $25 \mu \mathrm{M})$ for $1 \mathrm{~h}$ prior to treatment with FN for $48 \mathrm{~h}$. Cell migration and invasion, as well as processing of FAK, were subsequently analyzed. (A) The FN-induced cell migration was markedly suppressed by calpain inhibitors. (magnification, x100) (B) FN-induced cell invasion was significantly inhibited by ALLN, calpeptin and calpain inhibitor IV. (magnification, x100) (C) Pretreatment with calpain inhibitors suppressed FN-induced FAK processing. Values are expressed as the mean \pm standard error. "P<0.05, FN vs. FN+ALLN, FN+calpeptin and FN+calpain inhibitor IV. ALLN, calpain inhibitor I; cal IV, calpain IV; EMT, epithelial-mesenchymal transition; FAK, focal adhesion kinase 1; FN, fibronectin; con, control.

to being placed on FN. As shown in Fig. 4A, ALLN, calpeptin and calpain inhibitor IV caused a significant reduction in FN-induced migration. Furthermore, treatment with ALLN, calpeptin and calpain inhibitor IV inhibited FN-induced cell invasion (Fig. 4B). Whether FN-induced FAK cleavage was mediated via calpain activation was investigated. The results demonstrated that all three calpain inhibitors markedly blocked FN-induced FAK proteolytic cleavage, with significant downregulation of the $90 \mathrm{kDa}$ form (Fig. 4C). These data indicated that calpain has an important role in $\mathrm{FN}$-induced cell motility.

\section{Discussion}

According to Cancer Statistics 2014 (29), breast cancer is the most common type of cancer diagnosed and the second leading cause of cancer mortality among women. Metastasis is considered as one of the most important stages of tumor progression and remains responsible for $\sim 90 \%$ of patient mortalities, despite advances in the diagnosis and treatment of breast cancer (30). The process of tumor invasion and metastasis requires complex changes in cell-cell and cell-matrix interactions, thus apart from the accumulation of genetic and epigenetic changes in tumor cells, ECM components in the tumor microenvironment also have roles in tumor spreading, progression and therapeutic response (31). In recent years, a number of matrix components have been identified as important constituents of metastatic niches in breast cancer. Thus, performing an analysis of these ECM proteins and the associated signaling pathways is of enormous interest in the effort to identify therapeutic targets against advanced stages of breast cancer (1).

High levels of FN in the primary tumor have been linked to poor overall survival in breast cancer patients. In addition, increased expression of FN was also observed in lymph node metastases and was associated with an increased probability of metastasis $(3,32)$. Balanis et al (33) demonstrated that breast cancer cells at an early stage utilize Src-dependent epidermal growth factor (EGF) receptor signaling to promote the activation of signal transducer and activator of transcription 3 (STAT3). However, in metastatic breast cancer cells there is a switch to utilize FN-induced FAK/FAK2:JAK2:STAT3 signaling after cancer cells have acquired EMT, which indicates that a loss of responsiveness to growth factor is associated with an increase in the ability of FN to stimulate an alternative oncogenic pathway during metastatic progression. In addition, FN is able to increase the migratory ability and secretion of active matrix metalloproteinase-2 (MMP-2) in non-invasive MCF-7 breast cancer cells, and induce MMP-2 expression by decreasing its promoter methylation (34). In 
the present study, the ability of FN to promote cell migration and invasion as well as to induce the EMT progress in MCF-7 cells was also demonstrated. These findings indicate the important functional role of $\mathrm{FN}$ in breast cancer development, and its signaling pathway may be a potential target for novel therapeutic agents. This is consistent with a recent study that observed immunization against the alternatively spliced extra domain-A (ED-A) of FN by anti-ED-A antibody vaccination attenuates the progression of metastatic breast cancer (35).

Previously, studies have demonstrated that proteolytic activity of the calpain family regulates numerous intracellular proteins and is implicated in a variety of cellular processes, including cytoskeletal remodeling, cell adhesion, migration, proliferation and apoptosis (36). Additionally, calpain has been associated with metastatic potential of breast cancer both in vitro and in vivo $(15,16)$. The expression and activity of calpain is subject to complex regulation. Epidermal growth factor, $\mathrm{v}$-Src, the Ras signaling pathway, ERK 1/2 and estrogen can all stimulate the activity and expression of calpain (37). The present study demonstrated that calpain-2, which is involved in breast cancer cell migration and invasion $(19,38)$, was significantly upregulated following treatment with FN. It was further demonstrated that calpain inhibitors inhibited FN-induced migration and invasion of MCF-7 cells. Notably, treatment with FN caused no apparent changes in calpain-1 expression, indicating that FN may promote cell motility via calpain-2 activation. However, the underlying molecular mechanisms remain to be elucidated. FAK, a central component of focal adhesions, regulates cell-substrate attachment and cell motility. More notably, it is a specific substrate of calpain, which can be truncated into low molecular weight forms via calpain (39). The $30 \mathrm{kDa}$ C-terminal fragment of FAK contains the focal adhesion targeting sequence, which is required for association with other focal adhesion proteins, including breast cancer anti-estrogen resistance protein 1 , paxillin and talin (40). The $90 \mathrm{kDa}$ amino-terminal fragment is essential for kinase function and integrin-binding. The cleavage of FAK reduces its activity at cell adhesions, which leads to disassembling of focal adhesion complexes, loss of cell adhesion and increased cell motility (41). Previous studies have demonstrated that FAK is able to transduce FN-induced survival signals (42). Additionally, attachment of serum-starved MCF-10A cells to FN stimulates the activation of FAK-Src signaling (43). In the present study, in response to FN stimulation, the cleavage of FAK from $120 \mathrm{kDa}$ to $90 \mathrm{kDa}$ was increased. However the generation of a $90 \mathrm{kDa} F A K$ was significantly repressed by calpain inhibitors, which implied that modulation of FAK function through calpain-dependent cleavage is likely to play a significant role in the process of FN-induced motility.

EMT is a physiological phenomenon during embryonic development and tissue remodeling. Notably, EMT is essential for the development of cancer metastasis (44). The impaired expression of epithelial markers (E-cadherin and ZO-1), leads to the dissolution of cell adherence and tight junctions and an increase in the expression of mesenchymal markers (N-cadherin and vimentin), which are usually correlated with increased tumor migration and invasion (45). A wide range of factors from the micro-environment regulate this process, including TGF- $\beta$, tumor necrosis factor $\alpha$, and EGF. The results of the present study indicated that FN also induces an EMT response in MCF-7 breast cancer cells with a change in cell phenotype, including an increased expression of N-cadherin and vimentin as well as a decreased expression of E-cadherin and ZO-1 (Fig. 1). Notably, inhibition of calpain by calpain inhibitors suppressed FN-induced EMT in MCF-7 cells (Fig. 3), which suggests calpain is a requisite for FN-induced EMT response. However, the detailed mechanisms of how FN induces calpain remain to be elucidated.

In conclusion, the results of the present study demonstrated that FN enhances cell migration, invasion and the EMT process, which may facilitate metastatic progression of breast cancer. In addition, it has also been demonstrated that calpain has an essential role in the FN-induced EMT response. Targeting calpain may be a potential strategy to reduce breast cancer metastasis, and it would also be of great value to evaluate the effects of pharmacological calpain inhibitors on the treatment of breast cancer.

\section{Acknowledgements}

The present study was supported by the following funding bodies and programs: The Chinese National Natural Science Foundation (grant nos. 81560598,81402969 and 81302804), the Jiangsu Natural Science Foundation (grant no. BK20130220), the Chinese Postdoctoral Science Foundation (grant nos. 2013M541733 and 2015M582749XB), the Jiangsu Planned Projects for Postdoctoral Research Funds (grant no. 1301015B), the Science Foundation of Guiyang Science and Technology Bureau [grant no. (20141001) 06], the Science and Technology Innovation Advanced Individual of Guizhou Department of Education [grant no. QJHKY (2015) 492], the Guizhou Natural Science Foundation [grant no. QKHJ (2014) 2007], the Foundation for Training Programs of Innovation and Entrepreneurship for Undergraduates at the Guiyang Medical University (grant nos. 201610660042 and 201410660038), the Guizhou Innovated Team of the Education Department (grant no. 2014-31), the Guizhou Innovation team [grant no. (2015) 4025], the Program for New Century Excellent Talents in University (grant no. NCET-13-0747), the High level Innovation Talents (grant no. 2015-4029), the Fund of Innovation Team of Guizhou Province (grant no. 2015-4025), the Fund of Innovated Team of the Education Department of Guizhou Province (grant no. 2014-31) and the 2011 Modern Drug of Cooperation Innovation [grant no. (2013) 04].

\section{References}

1. Oskarsson T: Extracellular matrix components in breast cancer progression and metastasis. Breast 22 (Suppl 2): S66-S72, 2013.

2. Lu P, Weaver VM and Werb Z: The extracellular matrix: A dynamic niche in cancer progression. J Cell Biol 196: 395-406, 2012.

3. Ioachim E, Charchanti A, Briasoulis E, Karavasilis V, Tsanou H, Arvanitis DL, Agnantis NJ and Pavlidis N: Immunohistochemical expression of extracellular matrix components tenascin, fibronectin, collagen type IV and laminin in breast cancer: Their prognostic value and role in tumour invasion and progression. Eur J Cancer 38: 2362-2370, 2002.

4. Bae YK, Kim A, Kim MK, Choi JE, Kang SH and Lee SJ: Fibronectin expression in carcinoma cells correlates with tumor aggressiveness and poor clinical outcome in patients with invasive breast cancer. Hum Pathol 44: 2028-2037, 2013. 
5. Zhou Z, Qutaish M, Han Z, Schur RM, Liu Y, Wilson DL and Lu ZR: MRI detection of breast cancer micrometastases with a fibronectin-targeting contrast agent. Nat Commun 6: 7984, 2015.

6. Sceneay J, Smyth MJ and Möller A: The pre-metastatic niche: Finding common ground. Cancer Metastasis Rev 32: 449-464, 2013.

7. Barkan D, Green JE and Chambers AF: Extracellular matrix: A gatekeeper in the transition from dormancy to metastatic growth. Eur J Cancer 46: 1181-1188, 2010.

8. Hanahan D and Weinberg RA: Hallmarks of cancer: The next generation. Cell 144: 646-674, 2011

9. Kalluri R: EMT: When epithelial cells decide to become mesenchymal-like cells. J Clin Invest 119: 1417-1419, 2009.

10. Park J and Schwarzbauer JE: Mammary epithelial cell interactions with fibronectin stimulate epithelial-mesenchymal transition. Oncogene 33: 1649-1657, 2014.

11. Hood JL, Brooks WH and Roszman TL: Subcellular mobility of the calpain/calpastatin network: An organelle transient. Bioessays 28: 850-859, 2006.

12. Storr SJ, Carragher NO, Frame MC, Parr T and Martin SG: The calpain system and cancer. Nat Rev Cancer 11: 364-374, 2011.

13. Storr SJ, Thompson N, Pu X, Zhang Y and Martin SG: Calpain in breast cancer: Role in disease progression and treatment response. Pathobiology 82: 133-141, 2015.

14. Shiba E, Kambayashi JI, Sakon M, Kawasaki T, Kobayashi T, Koyama H, Yayoi E, Takatsuka Y and Takai SI: Ca\&sup2+;-dependent neutral protease (Calpain) activity in breast cancer tissue and estrogen receptor status. Breast Cancer 3 : 13-17, 1996.

15. Libertini SJ, Robinson BS, Dhillon NK, Glick D, George M, Dandekar S, Gregg JP, Sawai E and Mudryj M: Cyclin E both regulates and is regulated by calpain 2 , a protease associated with metastatic breast cancer phenotype. Cancer Res 65 10700-10708, 2005.

16. Storr SJ, Lee KW, Woolston CM, Safuan S, Green AR, Macmillan RD, Benhasouna A, Parr T, Ellis IO and Martin SG: Calpain system protein expression in basal-like and triple-negative invasive breast cancer. Ann Oncol 23: 2289-2296, 2012.

17. Franco SJ, Rodgers MA, Perrin BJ, Han J, Bennin DA, Critchley DR and Huttenlocher A: Calpain-mediated proteolysis of talin regulates adhesion dynamics. Nat Cell Biol 6: 977-983, 2004.

18. Chan KT, Bennin DA and Huttenlocher A: Regulation of adhesion dynamics by calpain-mediated proteolysis of focal adhesion kinase (FAK). J Biol Chem 285: 11418-11426, 2010.

19. Cortesio CL, Chan KT, Perrin BJ, Burton NO, Zhang S, Zhang ZY and Huttenlocher A: Calpain 2 and PTP1B function in a novel pathway with Src to regulate invadopodia dynamics and breast cancer cell invasion. J Cell Biol 180: 957-971, 2008.

20. Hoskin V, Szeto A, Ghaffari A, Greer PA, Côté GP and Elliott BE: Ezrin regulates focal adhesion and invadopodia dynamics by altering calpain activity to promote breast cancer cell invasion. Mol Biol Cell 26: 3464-3479, 2015.

21. Wells A, Kassis J, Solava J, Turner T and Lauffenburger DA Growth factor-induced cell motility in tumor invasion. Acta Oncol 41: 124-130, 2002

22. Keshamouni VG, Jagtap P, Michailidis G, Strahler JR, Kuick R, Reka AK, Papoulias P, Krishnapuram R, Srirangam A, Standiford TJ, et al: Temporal quantitative proteomics by iTRAQ 2D-LC-MS/MS and corresponding mRNA expression analysis identify post-transcriptional modulation of actin-cytoskeleton regulators during TGF-beta-induced epithelial-mesenchymal transition. J Proteome Res 8: 35-47, 2009.

23. Meng XN, Jin Y, Yu Y, Bai J, Liu GY, Zhu J, Zhao YZ, Wang Z, Chen F, Lee KY and Fu SB: Characterisation of fibronectin-mediated FAK signalling pathways in lung cancer cell migration and invasion. Br J Cancer 101: 327-334, 2009.

24. Li C, Zhao Y, Yang D, Yu Y, Guo H, Zhao Z, Zhang B and Yin X: Inhibitory effects of kaempferol on the invasion of human breast carcinoma cells by downregulating the expression and activity of matrix metalloproteinase-9. Biochem Cell Biol 93: 16-27, 2015.
25. Drasin DJ, Robin TP and Ford HL: Breast cancer epithelial-to-mesenchymal transition: Examining the functional consequences of plasticity. Breast Cancer Res 13: 226, 2011.

26. Franco SJ and Huttenlocher A: Regulating cell migration: Calpains make the cut. J Cell Sci 118: 3829-3838, 2005.

27. Frame MC, Fincham VJ, Carragher NO and Wyke JA: v-Src's hold over actin and cell adhesions. Nat Rev Mol Cell Biol 3: 233-245, 2002

28. Carragher NO, Levkau B, Ross R and Raines EW: Degraded collagen fragments promote rapid disassembly of smooth muscle focal adhesions that correlates with cleavage of pp125(FAK), paxillin and talin. J Cell Biol 147: 619-630, 1999.

29. Siegel R, Ma J, Zou Z and Jemal A: Cancer statistics, 2014. CA Cancer J Clin 64: 9-29, 2014.

30. Chaffer CL and Weinberg RA: A perspective on cancer cell metastasis. Science 331: 1559-1564, 2011.

31. Joyce JA: Therapeutic targeting of the tumor microenvironment. Cancer Cell 7: 513-520,2005.

32. Fernandez-Garcia B, Eiró N, Marín L, González-Reyes S, González LO, Lamelas ML and Vizoso FJ: Expression and prognostic significance of fibronectin and matrix metalloproteases in breast cancer metastasis. Histopathology 64: 512-522, 2014.

33. Balanis N, Wendt MK, Schiemann BJ, Wang Z, Schiemann WP and Carlin CR: Epithelial to mesenchymal transition promotes breast cancer progression via a fibronectin-dependent STAT3 signaling pathway. J Biol Chem 288: 17954-17967, 2013.

34. Pereira IT, Ramos EA, Costa ET, Camargo AA, Manica GC, Klassen LM, Chequin A, Braun-Prado K, Pedrosa Fde O, Souza EM, et al: Fibronectin affects transient MMP2 gene expression through DNA demethylation changes in non-invasive breast cancer cell lines. PLoS One 9: e105806, 2014.

35. Femel J, Huijbers EJ, Saupe F, Cedervall J, Zhang L, Roswall P, Larsson E, Olofsson H, Pietras K, Dimberg A, et al: Therapeutic vaccination against fibronectin ED-A attenuates progression of metastatic breast cancer. Oncotarget 5: 12418-12427, 2014.

36. Gora $\mathrm{J}$ and Latajka R: Involvement of cysteine proteases in cancer. Curr Med Chem 22: 944-957, 2015.

37. Leloup L and Wells A: Calpains as potential anti-cancer targets. Expert Opin Ther Targets 15: 309-323, 2011.

38. Ho WC, Pikor L, Gao Y, Elliott BE and Greer PA: Calpain 2 regulates Akt-FoxO-p27(Kip1) protein signaling pathway in mammary carcinoma. J Biol Chem 287: 15458-15465, 2012.

39. Goll DE, Thompson VF, Li H, Wei W and Cong J: The calpain system. Physiol Rev 83: 731-801, 2003.

40. Legate KR, Wickström SA and Fässler R: Genetic and cell biological analysis of integrin outside-in signaling. Genes Dev 23: 397-418, 2009.

41. Carragher NO, Fincham VJ, Riley D and Frame MC: Cleavage of focal adhesion kinase by different proteases during SRC-regulated transformation and apoptosis. Distinct roles for calpain and caspases. J Biol Chem 276: 4270-4275, 2001.

42. Ilic D, Almeida EA, Schlaepfer DD, Dazin P, Aizawa S and Damsky CH: Extracellular matrix survival signals transduced by focal adhesion kinase suppress p53-mediated apoptosis. J Cell Biol 143: 547-560, 1998

43. Kim NG and Gumbiner BM: Adhesion to fibronectin regulates Hippo signaling via the FAK-Src-PI3K pathway. J Cell Biol 210: 503-515, 2015.

44. Polyak K and Weinberg RA: Transitions between epithelial and mesenchymal states: Acquisition of malignant and stem cell traits. Nat Rev Cancer 9: 265-273, 2009.

45. Bedi U, Mishra VK, Wasilewski D, Scheel C and Johnsen SA: Epigenetic plasticity: A central regulator of epithelial-to-mesenchymal transition in cancer. Oncotarget 5: 2016-2029, 2014. 